\title{
CONCEPTOS BIOCLIMÁTICOS Y SU APLICABILIDAD A LA ZONA RURAL ALTOANDINA: CASO COMUNIDAD SAN FRANCISCO DE RAYMINA (SFR)-AYACUCHO
}

\author{
BIOCLIMATIC CONCEPTS AND THEIR APPLICABILITY TO \\ ANDEAN RURAL AREA: SAN FRANCISCO DE RAYMINA \\ COMMUNITY (SFR) CASE-AYACUCHO
}

\author{
Rafael Espinoza ${ }^{1}$, Jessica Molina², Manfred Horn ${ }^{3}$ Mónica M. Gómez ${ }^{4}$
}

RESUMEN

\begin{abstract}
Un adecuado tratamiento a la problemática que las viviendas rurales altoandinas atraviesan durante los meses de invierno, pasa por un acertado enfoque bioclimático. Así lo demuestra la intervención de la Universidad Nacional de Ingeniería, por medio del Centro de Energías Renovables (CER-UNI) y la Facultad de Ciencias, en la Comunidad San Francisco de Raymina (SFR), Ayacucho. En esta Comunidad se ha construido una vivienda bioclimática, mediante la cual se ha alcanzado un bienestar térmico a sus ocupantes. Este trabajo ha pasado por claramente establecer $y$, acertadamente, aplicar los conceptos bioclimáticos, algunos de los cuales son presentados en el presente artículo, así también como parte de la experiencia de la UNI en la Comunidad SFR.
\end{abstract}

Palabras clave.- Conceptos bioclimáticos, Zona rural altoandina, Heladas, Bienestar térmico.

\begin{abstract}
An appropriate treatment of the problems that people, living in high Andean rural areas, have during the winter months with their cold houses, passes through a correct bioclimatic approach. This is demonstrated with the involvement of the Centre for Renewable Energy and the Faculty of Science of the National University of Engineering (UNI) in San Francisco de Raymina community (SFR) in Ayacucho. In this community a bioclimatic house has been built, allowing a thermal comfort of the occupants. This work has been done applying clearly and correctly bioclimatic concepts, some of which are presented in this article, as well as part of the experience of UNI in SFR community.
\end{abstract}

Key words.- Bioclimatic concepts, Andean rural area, Frost, thermal wellness.

\section{INTRODUCCIÓN}

Las zonas rurales altoandinas con altitudes comprendidas entre 3000 y $5000 \mathrm{msnm}$ albergan en el Perú aproximadamente una población de 6 millones de personas, que cada año se encuentra expuesta a temperaturas mínimas que oscilan entre $-20^{\circ} \mathrm{C}$ y $-1{ }^{\circ} \mathrm{C}$. Esta información se observa claramente en la Figura 1, en la que se muestra el
Mapa de Heladas en el Perú [1], en el cual se da la información histórica del promedio de la temperatura mínima en diferentes partes del país durante los años 1964 y 2009.

En el mapa se observa que los departamentos más expuestos a temperaturas en el rango de $-25^{\circ} \mathrm{C}$ a $15^{\circ} \mathrm{C}$ son Puno, Cusco y Arequipa.

La Revista Científica TECNIA protege los derechos de autor bajo la Licencia 4.0 de Creative Commons: Attribution 4.0 International (CC BY 4.0)

${ }^{1} \mathrm{Mg}$. Director del CER-UNI. ${ }^{2}$ Bach. Asistente de investigación del CER-UNI. ${ }^{3}$ Dr. Investigador de la Facultad de Ciencias-UNI. ${ }^{4}$ Dra Investigadora de la Facultad de Ciencias-UNI. 
Temperaturas mínimas en el rango de $-15^{\circ} \mathrm{C}$ y $-10^{\circ} \mathrm{C}$ se presentan para los departamentos de Huánuco, Pasco, Junín, Huancavelica, Ayacucho, Apurímac, Cusco, Puno y Arequipa, en los cuales

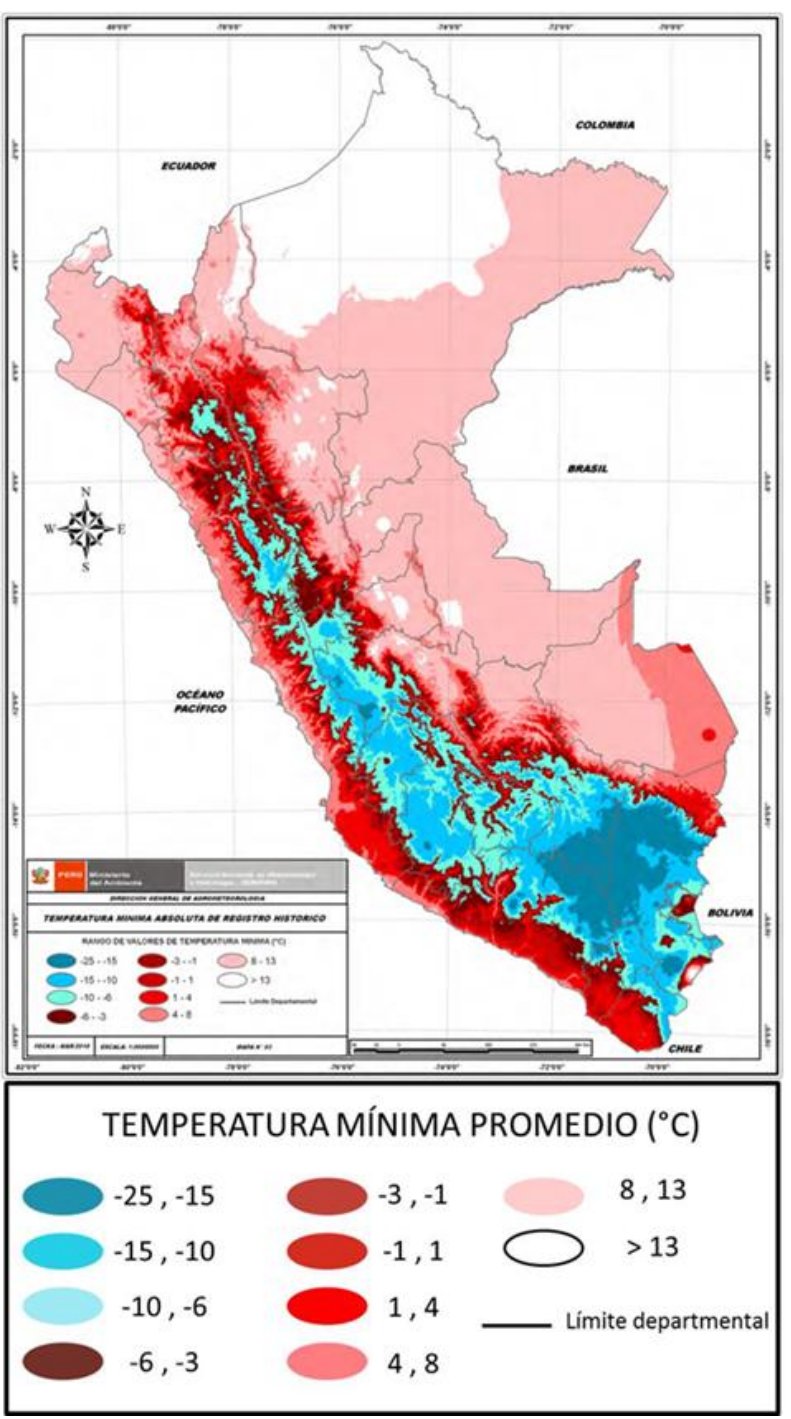

Fig. 1 Mapa del Perú con temperatura mínima absoluta de registro histórico (1964-2009) [1].

el número de días promedio de heladas durante los años 1964-2009 fue mayor a 60 por año, pudiendo llegar a 270 en departamentos como Junín, Huancavelica, Ayacucho, Cusco y Puno. Sin embargo esta condición natural contrasta con la disponibilidad de radiación solar que es especialmente abundante en la costa y sierra centro-sur del país, que en promedio está entre 6,0$6,5 \mathrm{kWh} /\left(\mathrm{m}^{2}\right.$ día), como se puede apreciar en la Figura 2, donde se presenta la insolación solar media anual en el Perú [2].
Los pobladores rurales en las zonas alto andinas que viven alejados de la ciudad, en su mayoría

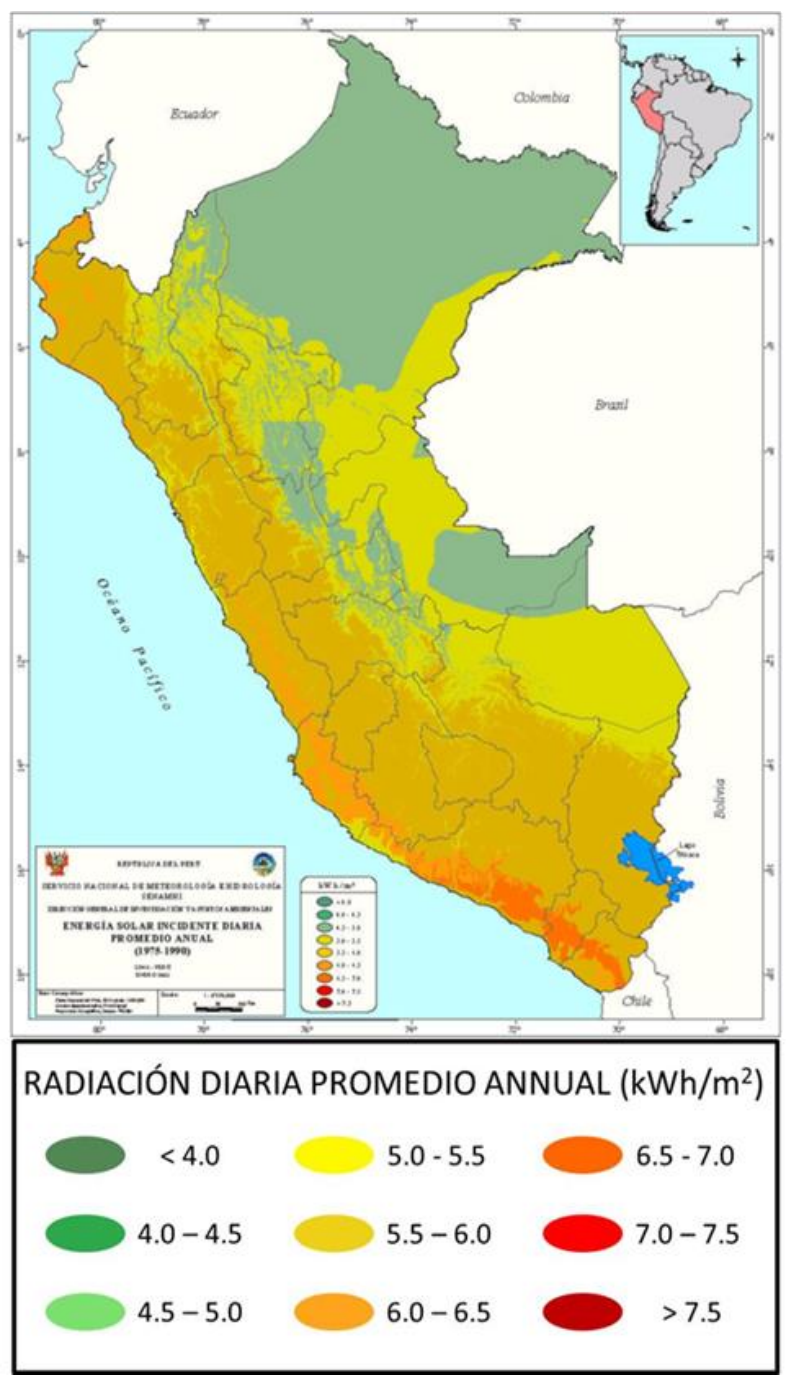

Fig. 2 Mapa del Perú para el promedio anual de energía solar incidente diaria [2].

no acceden a cubrir sus necesidades básicas de energía, entre las que destaca la de calefacción dentro de sus hogares. Según los datos del Instituto Nacional de Estadística e Informática (INEI) se sabe que la incidencia de pobreza se concentra en la zona rural del país, y que además los departamentos con mayores índices de pobreza extrema son Huancavelica, Huánuco, Apurímac, Ayacucho y Puno [3]. Las viviendas en estas zonas rurales alto andinas son precariamente construidas y no ofrecen a sus habitantes la protección térmica indispensable que resguarde su salud.

En los últimos años el Gobierno Central ha tomado atención a los graves problemas que cada año, 
durante los meses de invierno, las poblaciones de estas zonas rurales atraviesan. Lamentablemente todas las acciones, hasta el momento, se han quedado en un abordaje superficial que aún no alcanzan el análisis bioclimático que permita desarrollar adecuadamente soluciones sostenibles a futuro. Frente a esta situación, desde la Universidad Nacional de Ingeniería, por medio del Centro de Energía Renovables (CER-UNI) [4] y la Facultad de Ciencias [5], se vienen desarrollando esfuerzos por desarrollar un enfoque bioclimático a esta problemática.

En el presente artículo se presentan algunos conceptos que determinan un adecuado enfoque bioclimático, y se reporta la metodología desarrollada en la Comunidad San Francisco de Raymina (Ayacucho).

\section{Conceptos bioclimáticos}

Los conceptos bioclimáticos involucran varias disciplinas, como fisiología humana, climatología y física [6]. Una visión bioclimática se presenta como una conexión estrecha entre el hombre con la naturaleza, por ello se requieren datos específicos de ambas partes. Datos del grupo humano que se desarrolla en determinado ambiente $\mathrm{y}$, por otro lado, datos históricos y precisos del clima. A continuación, se presentan los conceptos más importantes usados en el enfoque bioclimático para la concepción de una edificación.

\section{Bienestar térmico (BT)}

También conocido como confort térmico, representa la comodidad que siente una persona dentro de un ambiente determinado durante el desempeño de sus actividades. Esta condición es el resultado complejo de la interacción de diversos factores entre los que se encuentran aspectos ambientales que rodean a la persona o aspectos propios de aquella, como son su condición física, su estado de salud, su vestimenta, su estado mental y hasta la influencia social [7]. Algunos de estos aspectos no son fácilmente medibles, pero hay otros que sí lo son, como es el caso de la temperatura ambiental, parámetro necesario para determinar la temperatura radiante ${ }^{1}$ del ambiente interior [8].

\section{Clima}

Definitivamente el clima es un aspecto ambiental determinante en el estado de confort térmico, y puede definirse como un conjunto de condiciones meteorológicas que se presentan en una determinada región. Por ello el clima puede describirse por medio de sus elementos constituyentes, entre lo que destacan la temperatura y las precipitaciones; así como por los factores que lo modifican, que pueden presentarse relacionados por el grado de insolación, que a su vez está ligado con la ubicación geográfica. La Figura 3 muestra un esquema con los elementos que constituyen el clima, así como los factores que lo modifican.

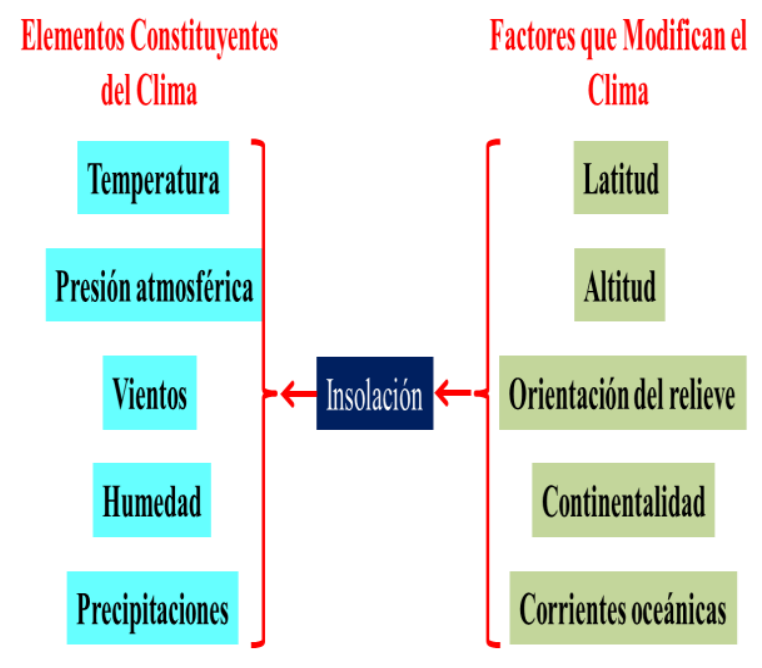

Fig. 3 Representación de los elementos constituyentes del clima y factores que pueden modificarlo [9].

\section{Índices bioclimáticos (IB)}

Nacen del tratamiento estadístico de la información brindada por un grupo humano específico que manifiesta lo que siente en determinado ambiente. Mediante la definición de

\footnotetext{
1 Se denomina a la temperatura que adquiere una persona en el interior de un ambiente, la que depende no solamente de la temperatura del aire en el ambiente, sino también de otros factores, como la temperatura y propiedades de las paredes, etc.
} 
los IB se puede cuantificar la sensación de bienestar o desconformidad de la población a la cual va orientada el objetivo de aplicar un proyecto bioclimático.

Mediante la definición de los IB se puede generar lo que se conoce como la zona de confort, la cual brinda información del intervalo de temperatura y humedad en la cual una persona, perteneciente al grupo humano evaluado, sentirá BT. La determinación de la zona de confort tiene un carácter predictivo y cuantificable. Así estos IB se pueden presentar mediante ecuaciones $\mathrm{o}$ diagramas.

Es por ello de gran importancia determinar los IB para el caso específico de los pobladores de las zonas rurales altoandinas, trabajo que aún no sea realizado. Por ello los trabajos bioclimáticos hasta ahora desarrollados emplean IB tomados de otras zonas del planeta.

\section{Estrategias bioclimáticas (EB)}

Se designan así al conjunto de procedimientos de adecuación de una vivienda que tienen en cuenta las condiciones climáticas y la interacción de esta con el medio ambiente. Ellas son, básicamente, las normas generales que tienen por objeto orientar el diseño de la vivienda aprovechando las condiciones meteorológicas de cada lugar. Las EB pueden acercar la vivienda al BT y reducir así su consumo energético [10].

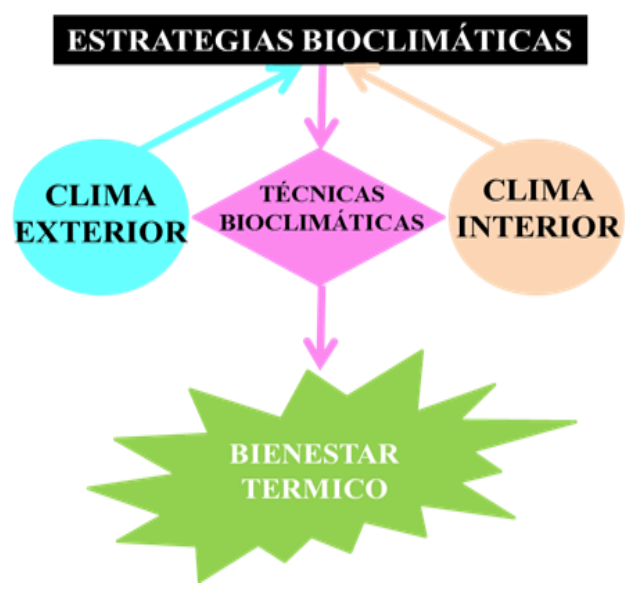

Fig.4 Representación del proceso de determinación de estrategias bioclimáticas y su relación con las técnicas bioclimáticas y el bienestar térmico [9].
También conocidas como componentes bioclimáticos es el camino a seguir para poder aplicar las EB.

Se puede afirmar entonces que el uso de IB, para determinado ambiente, define el conjunto de EB a ser empleadas para alcanzar el BT de las personas que desarrollaran sus actividades dentro del ambiente en mención. La adecuada interpretación de las EB generará TB que deberán ser aplicadas en una determinada edificación para que esta pueda brindar bienestar térmico a sus ocupantes.

\section{PARTE EXPERIMENTAL}

\section{Comunidad San Francisco de Raymina (SFR)- Ayacucho}

La Comunidad de SFR se encuentra ubicada en el distrito de Huamballpa, provincia de Vilcas Huamán-Ayacucho. Actualmente en esta comunidad viven 40 familias, que tienen sus viviendas en una zona ubicada a $3700 \mathrm{msnm}$, y su área de cultiva se encuentra a 2800 - $3800 \mathrm{msnm}$. La tercera parte de sus habitantes no tienen una permanencia constante en la comunidad.

La temporada de lluvias es entre diciembre y abril, y entre los meses de abril a noviembre el clima es frio y soleado. Se presentan ocasionalmente tormentas eléctricas y granizadas. Sus viviendas están sobre terrenos lotizados de, aproximadamente, $350 \mathrm{~m}^{2}$ cada uno.

La comunidad cuenta con una plaza principal, área de recreación pública, áreas de circulación consideradas como calles. Además, se cuenta con un colegio y un salón comunal construidos con material noble, equipados solo con mesas y sillas, y que, por sus características constructivas, en su interior son fríos, lo que naturalmente genera un malestar en los pobladores. Por ejemplo, los niños manifiestan su incomodidad al estudiar. El salón comunal es utilizado única y exclusivamente para reuniones y cursos de capacitación, pero en época de extremo frío no es utilizado. 


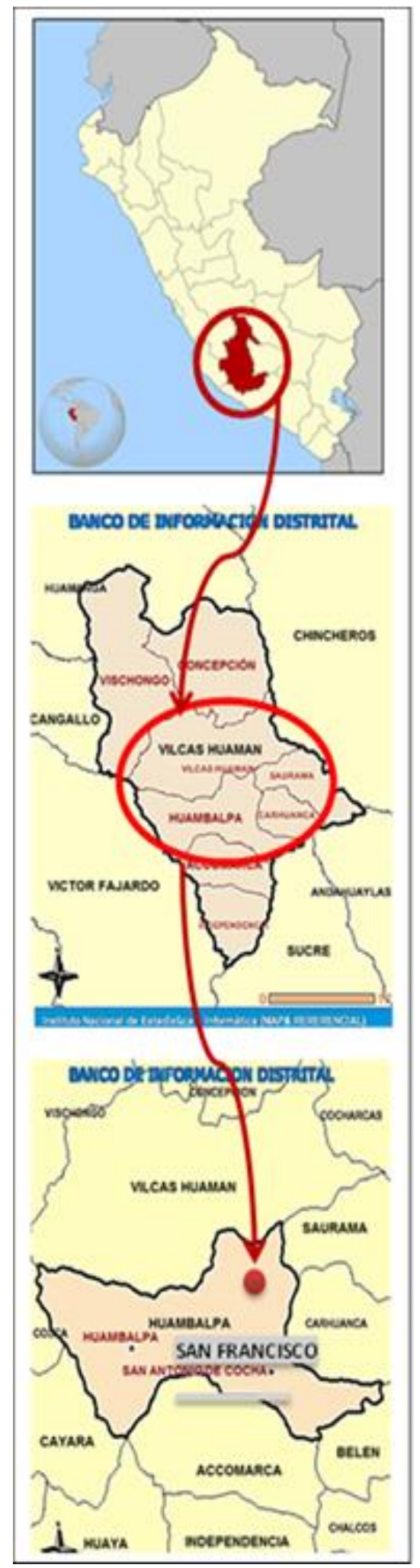

Fig.5 Ubicación geográfica de la Comunidad San Francisco de Raymina-Ayacucho [9].

\section{INTERVENCION DE LA UNI EN LA COMUNIDAD SFR}

El recurso solar es la principal fuente que permitiría energizar las poblaciones más afectadas por las heladas, por ello es muy importante conocer en detalle el recorrido del sol durante todo el año, sobre el poblado en estudio. La Figura 6 muestra esta información para la Comunidad SFR, donde cada línea azul y verde representa un día, y las líneas rojas muestran la hora solar. El sol se desplaza de este a oeste durante todo el año, en los meses de abril a setiembre está orientado hacia el norte de la comunidad, mientras que para los meses de octubre a febrero, lo está hacia el sur.

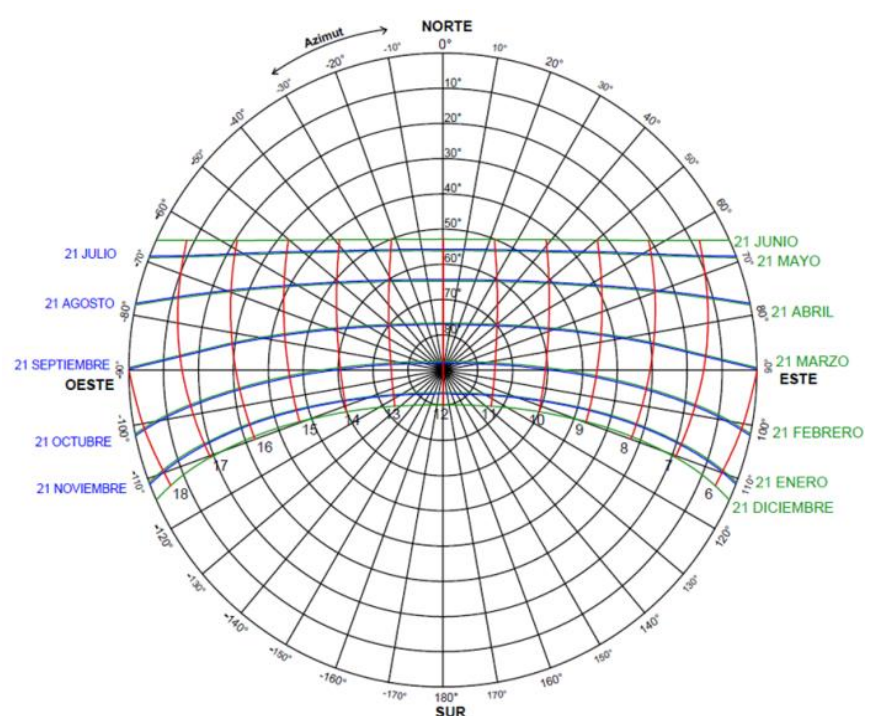

Fig. 6 Carta solar estereográfica de SRF (Latitud: $13^{\circ} 45^{\prime} 40^{\prime \prime} S$ y Longitud: $73^{\circ} 51$ '26'ㅇ) [11].

El Gobierno del Perú por medio del Ministerio de Vivienda, Construcción y Saneamiento (MVCS) junto con Organismos No Gubernamentales (ONGs), han ejecutado diversos programas en viviendas muy vulnerables a las heladas [12], en las cuales directamente han implementado TB sin mayor análisis antes de su implementación ni evaluación post-instalación. 
La intervención de la UNI en la Comunidad de SFR ha pasado por monitorear en detalle la información de las características del clima, información que no puede ser presentada en esta publicación por su extensión ${ }^{2}$.

Tampoco se podrán presentar en detalle todas las TB empleadas en las viviendas bioclimáticas construidas y modificadas en SFR, entre las que figuran [13]:

- Aislamiento térmico en pisos, techos y ventanas

- Eliminación de puentes térmicos

- Ventanas térmicas en techos (ganancia directa de la radiación solar durante el día)

- Muros de adobe con alta masa térmica y dispositivos radiantes (almacenamiento de calor para la noche)

- Invernaderos adosados a muros de zonas térmicas críticas

A continuación se presentaran dos TB muy importantes como es el invernadero adosado a una habitación, por medio del cual se permite el ingreso de aire caliente al interior de la vivienda, y la salida del aire frio en dirección al invernadero. El esquema de esta forma de ganancia indirecta de energía solar se muestra en la Figura 7.

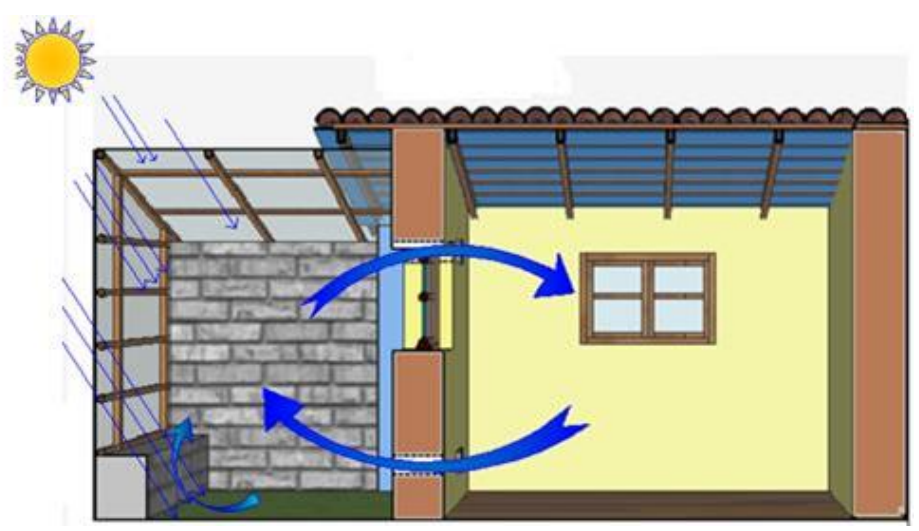

Fig. 7 Forma de ganancia indirecta [11].

Esta TB presenta doble beneficio a los ocupantes de la vivienda, no solo el relacionado al BT, sino

\footnotetext{
${ }^{2}$ Los trabajos de la UNI en SFR están descritos en detalle en las referencias 9 y 11 .
}

también el aspecto nutricional, dado que el invernadero permite el cultivo de plantas y hortalizas que son un importante aporte alimenticio para una población con difícil acceso a la ciudad y, que por el clima, su cultivo en el campo se ve limitado a ciertos productos.

Otro aspecto muy importante es el relacionado al aislamiento higrotérmico de la vivienda (aislamiento que evite el ingreso de agua y el intercambio de calor). Por ejemplo para el aislamiento de pisos, la TB empleada consistió en aplanar el piso de tierra, colocar una cama de piedras, listones de madera y sobre estos, un piso machihembrado de madera que se encuentra, además, a una distancia de las piedras, para dejar que el aire contribuya con el aislamiento del piso. La Figura 8 muestra un esquema de esta tipo de piso.

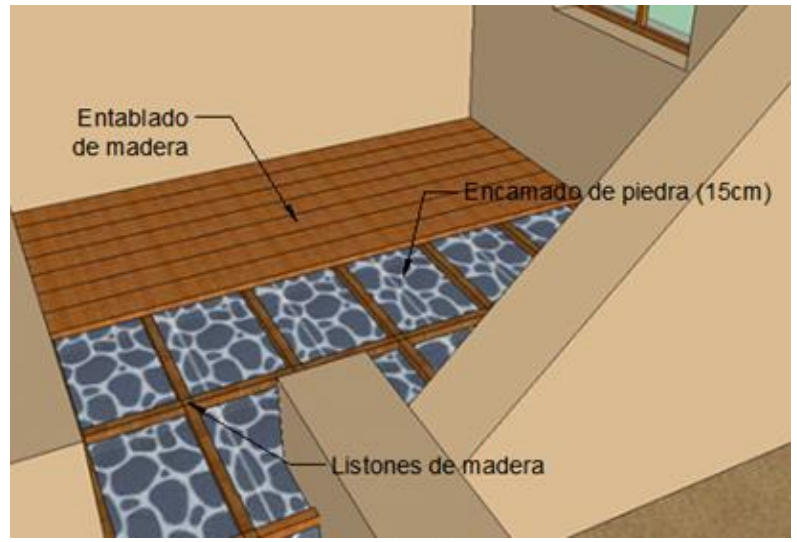

Fig. 8 Esquema del aislamiento higrotérmico de pisos [11].

Una imagen de una de las viviendas bioclimáticas en SFR se muestra la Figura 9. La cual cuenta aislamiento en el techo, constituido por capas de múltiples materiales como: madera, ichu, plástico y tejas. Además, en ciertas zonas cuenta con superficies de planchas de policarbonato que permiten la ganancia directa de la energía solar, esencial para alcanzar condiciones de bienestar térmico durante las $24 \mathrm{~h}$ del día.

Se observa también una esclusa (construida de manera similar que el invernadero) en la entrada principal de la vivienda, lo que impide un intercambio directo de aire entre el exterior y el interior. 


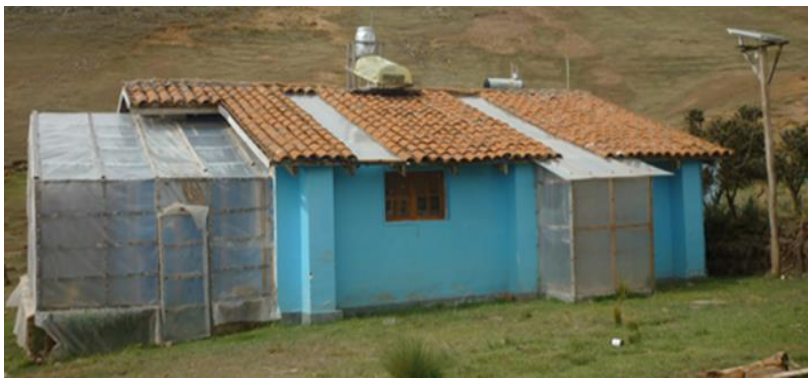

Fig.9 Vivienda rural bioclimática experimental construida en SFR.

Destacan en la parte superficial del techo un colector fototérmico (para el suministro de agua caliente) y, muy cerca, al lado derecho un módulo fotovoltaico (para suministro eléctrico).

\section{DISCUSIONES}

Las soluciones y las propuestas relacionadas a las variables ambientales deben incorporar beneficios de los aspectos favorables del clima, así como ofrecer protección de los elementos no favorables

Si bien es cierto que las propuestas técnicas deben presentarse íntimamente ligadas con el quehacer cotidiano de la comunidad, también se debe considerar el programa intensivo de capacitación en la comunidad que alcance no solo a los responsables directos de las viviendas, sino que además se implemente la capacitación a nivel escolar para que la población más joven cuente con conocimientos prácticos del uso de técnicas bioclimáticas en sus viviendas.

La intervención del CER-UNI en la Comunidad SFR ha considerado aspectos técnicos que fundamentan la aplicabilidad de aspectos bioclimáticos indispensables para enfrentar las temperaturas bajas extremas en los meses de invierno.

\section{CONCLUSIONES}

La adopción de principios bioclimáticos en el diseño de las casas ubicadas en zonas rurales altoandinas alejadas de la ciudad, se presenta como un camino sustentable y sostenible ante la problemática que la población ubicada en estos lugares atraviesa durante la época de heladas. El Gobierno Central no solo debe fomentar la implementación de técnicas bioclimáticas, en esas zonas tan vulnerables, sino también fortalecer la investigación básica y aplicada en el desarrollo adecuado de un enfoque bioclimático que brinde un soporte sólido a todo el trabajo que se realice en este ámbito. Un ejemplo de ello lo demuestra el trabajo desarrollado en la Comunidad SFR, mediante la financiación de proyectos de investigación que ha recibido la UNI en el área bioclimática. Esta condición es elemental para que las técnicas y conceptos bioclimáticos sean entendidos y aplicados adecuadamente. Esa visión evitaría gastos inútiles en proyectos ejecutados, como el caso de la instalación de los "Muros Trombe" durante los años 2009-2012 [14, 15] que no contó con ningún soporte técnico, y por ende representaron un fracaso económico y social para el país.

\section{AGRADECIMIENTOS}

Uno de nosotros (M.M.G.) agradece al Instituto General de Investigación de la Universidad Nacional de Ingeniería (IGI-UNI) por la financiación individual otorgada. El presente trabajo fue desarrollado bajo el auspicio del IGIUNI y el Consejo Nacional de Ciencia, Tecnología e Innovación Tecnología del Perú - CONCYTEC (Contratos 239-2011, 406-2012, 040-2013).

\section{BIBLIOGRAFÍA}

1. Senamhi and FAO, "Atlas de heladas del Perú - convenio de cooperación técnica interinstitucional" SENAMHI-FAO. 2010, Lima.

2. Senamhi, "Atlas de energía solar del Perú Proyecto PER/98/G31: Electrificación rural a base de energía fotovoltaica en el Perú". 2003, MEM: Lima. p. 31.

3. INEI, "Evolución de la Pobreza al 2009. 2010", Instituto Nacional de Estadística e Informática: Lima.

4. CER-UNI. Centro de Energías Renovables de la Universidad Nacional de Ingeniería. [cited 2015; Available from: http://cer.uni.edu.pe/.

5. FC. Posgrado: Planes de Estudio. 2016 [cited 2016; Available from: http://fc.uni.edu.pe/fc/index.php/posgrado/linea s-disponibles.

6. Olgyay, V. and A. Olgyay, "Design with Climate: Bioclimatic Approach to Architectural Regionalism", ed. P.U. Press. 1963. 190. 
7. Givoni, B., "Climate Considerations in Building and Urban Design" 1ed. 1998, New York: Wiley.

8. Morillón, D., et al., "Atlas Bioclimático de la República Mexicana”. Energías Renovables y Medio Ambiente, 2002. 10: p. 57-62.

9. Espinoza Paredes, R.L., "Evaluación experimental de la performance de dos componentes bioclimáticas de calentamiento aplicadas en un módulo de vivienda de San Francisco de Raymina-Ayacucho con el propósito de validarlas como técnicas de estrategias bioclimáticas para viviendas rurales alto andina", Tesis de Maestría, Facultad de Ciencias. 2014, Universidad Nacional de Ingeniería: Lima.

10. Goncalçves, H. and J. Mariz Graça, "Conceitos bioclimáticos para os edificios em Portugal". 2004: Lisboa.

11. Molina, J., "Evaluación bioclimática de una vivienda rural alto andina de la Comunidad de San Francisco de Raymina de Ayacucho", Tesis de Maestría, Facultad de Ciencias. 2016, Universidad Nacional de Ingeniería: Lima.

12. GIZ-MVCS, "Abrigando hogares experiencias con medidas de confort térmico en viviendas rurales altoandinas". 2015, Lima.

13. Gómez, M.M., R. Espinoza, and M. Horn, "Energy for unserved populations, in Guide towards a sustainable energy future for the Americas", IANAS, Editor. 2016, IANAS-IAP: México. p. 38-61.

14. SENCICO, "Resolución de General in 0432009-03.00, SENCICO”, Editor. 2009, SENCICO: Lima. p. 28.

15. SENCICO-MVCS, "Calefactor Solar SENCICO: Responsabilidad Social, in Suplementos Especiales de Construcción y Vivienda”. 2009, SENCICO: Lima.

Correspondencia: mgomez@uni.edu.pe 